\title{
PCDHIO Promoter Hypermethylation is Frequent in most Histologic Subtypes of Mature Lymphoid Malignancies and Occurs Early in Lymphomagenesis
}

\author{
Gopeshwar Narayan, ${ }^{\dagger \dagger}$ Dongxu Xie, ${ }^{2}$ Allen J. Freddy,, Ganchimeg Ishdorj, ${ }^{2}$ Catherine Do, ${ }^{2}$ Prakash Satwani, ${ }^{3}$ \\ Hema Liyanage, ${ }^{4}$ Lorraine Clark, ${ }^{\prime}$ Sergey Kisselev, 'Subhadra V. Nandula, ${ }^{\prime \neq}$ Luigi Scotto, ${ }^{5}$ Bachir Alobeid, \\ David Savage, ${ }^{5}$ Benjamin Tycko, ${ }^{1,2}$ Owen A. O'Connor, ${ }^{5}$ Govind Bhagat,' and Vundavalli V. Murty ${ }^{1,2 *}$ \\ 'Department of Pathology and Cell Biology, Columbia University Medical Center, New York Presbyterian Hospital, New York, NY \\ ${ }^{2}$ Institute for Cancer Genetics, Irving Cancer Research Center, Columbia University Medical Center, New York, NY \\ ${ }^{3}$ Department of Pediatrics, Columbia University Medical Center, New York, NY \\ ${ }^{4}$ Applications and Technology Division, Sequenom, Inc. San Diego, CA \\ ${ }^{5}$ Department of Medicine, Columbia University Medical Center, New York, NY
}

PCDHIO is epigenetically inactivated in multiple tumor types; however, studies in mature lymphoid malignancies are limited. Here, we have investigated the presence of promoter hypermethylation of the PCDHIO gene in a large cohort of well-characterized subsets of lymphomas. PCDHIO promoter hypermethylation was identified by methylation-specific PCR in 57 to $100 \%$ of both primary B- and T-cell lymphoma specimens and cell lines. These findings were further validated by Sequenom Mass-array analysis. Promoter hypermethylation was also identified in $28.6 \%$ cases of reactive follicular hyperplasia, more commonly occurring in states of immune deregulation and associated with rare presence of clonal karyotypic aberrations, suggesting that PCDHIO methylation occurs early in lymphomagenesis. PCDHIO expression was down regulated via promoter hypermethylation in T- and B-cell lymphoma cell lines. The transcriptional down-regulation resulting from PCDHIO methylation could be restored by pharmacologic inhibition of DNA methyltransferases in cell lines. Both Tand B-cell lymphoma cell lines harboring methylation-mediated inactivation of PCDHIO were resistant to doxorubicin treatment, suggesting that hypermethylation of this gene might contribute to chemotherapy response. (C) 2013 Wiley Periodicals, Inc.

\section{INTRODUCTION}

The protocadherin subfamily of the cadherin superfamily of genes that encode cadherin-related neuronal receptors plays a role in the establishment and function of specific cell-cell connections (Yagi, 2008). PCDH10, a member of this family, has been implicated as tumor suppressor gene by epigenetic inactivation in multiple human cancer types (Ying et al., 2006, 2007; Narayan et al., 2009; Yu et al., 2009; Cheung et al., 2010) and promoter $\mathrm{CpG}$ island hypermethylation-associated PCDH10 gene silencing is a frequent event in myeloid and lymphoid leukemias (Garcia-Manero et al., 2009; Narayan et al., 2011). However, data regarding the role of PCDH10 in mature lymphoid malignancies are limited. Few reports have shown high frequency of PCDH10 methylation and associated down-regulation of expression in a limited panel of $\mathrm{T}$ - and B-cell lymphoma cell lines and primary tumors (Ying et al., 2007; Li et al., 2012).

Epigenetic processes, most notably patterns of DNA promoter sequence hypermethylation, play a central role in cellular transformation and treatment of cancer. Specific genetic mutations that confer chromatin modifications at certain loci have led to the development of therapies influencing the epigenetic landscape in leukemia (Nowak et al., 2009; Baylin and Jones, 2011). Although similar advances in malignant lymphoma are lacking, recently there has been an intense growing interest in understanding the role of epigenetic modifications in lymphoid malignancies (Zain and O’Connor, 2010; Seton-Rogers, 2012).

Additional Supporting Information may be found in the online version of this article.

*Correspondence to: Vundavalli V. Murty, Irving Cancer Research Center, Room 605, Columbia University Medical Center, 1130 St. Nicholas Avenue, New York, NY 10032, USA. E-mail:vvm2@columbia.edu

Supported by: Lance Armstrong Foundation (to V.V.M.).

$\uparrow$ Present address: Banares Hindu University, Varanasi, India.

$\$$ Present address: Cancer Genetics, Inc, Rutherford, NJ.

Received 17 March 2013; Accepted 7 July 2013

DOI 10.1002/gcc.22098

Published online 9 August 2013 in

Wiley Online Library (wileyonlinelibrary.com) 
Here, we have examined the presence of promoter hypermethylation of $P C D H 10$ in various histologic subtypes of non-Hodgkin lymphoma (NHL) and found high frequency methylation in most lymphoma cases. Promoter hypermethylation resulted in down-regulated expression of $\mathrm{PCDH} 10$ in lymphoma cell lines, and cell lines carrying PCDH1O promoter methylation or down-regulated expression exhibited resistance to doxorubicin treatment. Furthermore, the presence of PCDH1O hypermethylation in a significant fraction of reactive lymph nodes suggests that promoter methylation might be an early event in lymphomagenesis.

\section{MATERIALS AND METHODS}

\section{Patient Samples and Cell Lines}

A total of 292 DNA samples from 291 patients derived from primary tumors diagnosed at our institute over a 12-year period (July 1997-June 2009) were analyzed, which represented 21 reactive lymph (RL) nodes exhibiting follicular hyperplasia $(\mathrm{FH})$, 50 chronic lymphocytic leukemia/small lymphocytic lymphomas (CLL/SLL), 19 Burkitt lymphomas (BL), 39 follicular lymphomas (FL), 41 diffuse large B-cell lymphomas (DLBCL), 38 marginal zone lymphomas (MZL), 16 mantle cell lymphomas (MCL), 35 multiple myelomas (MM), and 33 T/NK-cell lymphomas. All specimens used in this study were evaluated by morphology, flow cytometry, and cytogenetic analyses (Supporting Information Table 1) and classified according to the current WHO criteria (Swerdlow et al., 2008). All lymphoma cases were selected to obtain $>40 \%$ tumor cells either by morphology and/or fluorescence in situ hybridization (FISH) positivity. In addition, $10 \mathrm{~T}$-cell lymphoma cell lines (MT1, MT2, ED, CJ/M5, TLom1, SLB1, HH, H9, HUT-78, and FE-PD) and 10 B-cell lymphoma cell lines (Raji, Daudi, LY-1, LY-3, SU-DHL-4, SU-DHL-5, SU-DHL-8, SU-DHL-10, WSU, and Farage) (kindly provided by Riccardo Dalla-Favera, Columbia University, New York) were also utilized. All cell lines were grown in RPMI-1640 or IMDM medium containing 10 to $15 \%$ fetal bovine serum. Our institutional review board approved the study protocol.

\section{DNA and RNA Isolation}

Frozen specimens and cell lines were utilized for isolation of high molecular weight DNA and RNA by standard methods. RNA quality and quantity was assessed by a bioanalyzer (Agilent Technologies, Foster City, CA).

\section{Methylation Specific PCR (MSP)}

Two micrograms of genomic DNA was converted using EpiTect 96 bisulfite kit (Qiagen, Valencia, CA). Placental DNA treated in vitro with SssI methyltransferase (New England BioLabs, Beverly, MA) and normal lymphocyte DNA converted with sodium bisulfite was used as methylated and unmethylated controls, respectively. Four sets of primers utilized for amplification of methylated (M) DNA and two sets of primers for unmethylated (U) DNA spanning two $\mathrm{CpG}$ islands (CGIs) of cDNA clone NM-032961 were described previously (Narayan et al., 2009, 2011). PCR was performed using standard conditions for 30 35 cycles with annealing temperatures varying between 56 and $62^{\circ} \mathrm{C}$. All MSP experiments were performed in triplicate and promoter hypermethylation was considered positive when present in at least one of the regions in replicate experiments.

\section{Sequenom EpiTyper Quantitative Methylation Analysis}

Quantitative methylation analysis was performed using the Sequenom MALDI-TOF mass spectrometry platform as per manufacturer specifications (Sequenom, Inc. San Diego, CA) and as described previously (Narayan et al., 2011). Heat maps were generated by using either R-script or JMP genomics software (http://www.jmp.com/software/genomics/).

\section{Drug Treatment}

Cells in culture were treated with $5 \mu \mathrm{M}$ 5-aza$2^{\prime}$ deoxycytidine (5-aza-CdR) for 5 days by replacing the medium everyday, trichostatin (TSA) at a final concentration of $200 \mathrm{nM}$ for $24 \mathrm{hr}$ and combination of both as previously described (Narayan et al., 2009). Cells collected from these experiments were used for isolating total RNA. We determined ICD50 values for all NHL cell lines for the drugs used and the following final concentrations were used in the present experiments: doxorubicin $(15 \mathrm{ng} / \mathrm{ml})$, dexamethasone $(100 \mathrm{nM})$, bortezomib (2 $\mathrm{ng} / \mathrm{ml})$, methotrexate $(50 \mathrm{nM})$, and L-asparaginase $(2 \mathrm{U} / \mathrm{ml})$. Cell lines were treated with these drugs for $48 \mathrm{hr}$ to assess cell viability and apoptosis.

\section{Reverse Transcription PCR (RT-PCR) and Western Blot Analysis}

Total RNA isolated from cell lines was reverse transcribed as described (Narayan et al., 2009). Semiquantitative expression of $\mathrm{PCDH10}$ was 
performed in triplicate RT-PCR experiments using the primers described previously and standard thermal cycle conditions (Narayan et al., 2011). The $\beta$-actin gene was used as control to assess the relative intensity of mRNA expression. Western blot analysis was performed by standard methods using polyclonal PCDH10 antibody (Thermo Scientific, Rockford, IL) and a secondary antibody conjugated with horseradish peroxidase (Bio-Rad, Hercules, CA). Detection was performed by ECL-Western Lightning Chemiluminescence reagent (Amersham Pharmacia) and the blot was re-probed with $\beta$-actin as control.

\section{Cell Viability and Apoptosis Assays}

Cell viability and cytotoxicity due to each drug was assessed by standard MTT (3-(4,5-dimethylthiazol-2-yl)-2,5-diphenyltetrazolium bromide) (Invitrogen, Carlsbad, CA) colorimetric assay. Briefly, 5,000 cells were seeded in 96-well cell culture plates, grown overnight at $37^{\circ} \mathrm{C}$ in $5 \%$ $\mathrm{CO}_{2}$ incubator, and treated with drugs for $48 \mathrm{hr}$. After incubation of cells in $5 \mathrm{mg} / \mathrm{ml}$ of MTT in phosphate buffer saline for $3.5 \mathrm{hr}$, medium was removed, the dye was dissolved in $150 \mu$ of MTT solvent and the optical density of solubilized formazan was assessed using a microplate reader (BioTek Quant, Winooski, VT). All treatments were performed in four replicate wells in two independent experiments. To measure apoptosis, we used Pacific Blue ${ }^{\mathrm{TM}}$ Annexin V/SYTOX ${ }^{\circledR}$ AADvanced $^{\text {TM }}$ Apoptosis Kit (Invitrogen). Briefly, 0.5 to 1.0 million cells were seeded in 48 -well tissue culture plates, grown overnight at $37^{\circ} \mathrm{C}$ at $5 \% \mathrm{CO}_{2}$ incubator, and the indicated concentration of drugs was added. Cells collected after $48 \mathrm{hr}$ of incubation were analyzed by a LSR II flow cytometer (BD Biosciences, San Jose, CA) using $405 \mathrm{~nm}$ and $488 \mathrm{~nm}$ excitation and $455 \mathrm{~nm}$ and $647 \mathrm{~nm}$ emissions. A total of 20,000 events were analyzed using Flowjo software (Tree Star, OR) in duplicate experiments.

\section{Karyotype and Fluorescence In Situ Hybridization (FISH) Analyses}

G-band karyotype and FISH analyses were performed using standard methods.

\section{Statistical Analysis}

ANOVA and $t$-test statistics were calculated using the GraphPad Prism software (LaJolla, CA).
TABLE I. Frequency of $P C D H I O$ Promoter Hypermethylation in Mature B- and T-Cell Lymphomas

\begin{tabular}{lcc}
\hline Histologic subtype & $\begin{array}{c}\text { No. } \\
\text { Studied }\end{array}$ & $\begin{array}{c}\text { Methylated } \\
(\%)\end{array}$ \\
\hline $\begin{array}{l}\text { Reactive lymph node/follicular } \\
\text { hyperplasia }\end{array}$ & 21 & $6(29 \%)$ \\
$\begin{array}{l}\text { Chronic lymphocytic leukemia } \\
\text { Burkitt's lymphoma }\end{array}$ & 50 & $33(66 \%)$ \\
Follicular lymphoma & 19 & $18(95 \%)$ \\
Diffuse large B-cell lymphoma & 39 & $39(100 \%)$ \\
Marginal zone lymphoma & 41 & $41(100 \%)$ \\
Mantle cell lymphoma & 38 & $34(90 \%)$ \\
Multiple myeloma & 16 & $15(94 \%)$ \\
Natural killer (NK)/T-cell lymphoma & 35 & $20(57 \%)$ \\
\hline
\end{tabular}

\section{RESULTS}

MSP Analysis Identifies High Frequency of PCDHIO Promoter hypermethylation in Various Histologic Subtypes of Non-Hodgkin Lymphomas and Multiple Myeloma

The PCDH10 gene promoter region spanning two classic $\mathrm{CpG}$ islands was examined by MSP for qualitative assessment of methylation as previously described (Supporting Information Fig. 1A) (Narayan et al., 2011). Promoter methylation analysis was performed on a total of 292 DNA samples derived from various histologic subtypes of B- and T-cell lymphomas (Table 1). Among the B-cell NHL, promoter hypermethylation was found in all $39(100 \%) \mathrm{FL}$ and $41(100 \%)$ DLBCL, $18(95 \%)$ of $19 \mathrm{BL}, 15(94 \%)$ of $16 \mathrm{MCL}, 34(90 \%)$ of 38 MZL, $33(66 \%)$ of 50 CLL, and $20(57 \%)$ of 35 MM samples (Table 1). Among the $33 \mathrm{~T}$ - and NK-cell lymphomas examined, 26 (79\%) showed promoter hypermethylation (Table 1). Similar analysis on DNA isolated from 21 specimens of reactive follicular hyperplasia revealed hypermethylation in six $(29 \%)$ cases. Of note, 11 of these cases occurred in individuals with known immune deregulation and five displayed minor clonal karyotypic abnormalities. Therefore, these data provide evidence that a large majority of cases across all major histologic subtypes of lymphomas harbor PCDH10 promoter hypermethylation and suggest that inactivation of this gene may be related to disease pathogenesis. The presence of promoter hypermethylation in reactive lymph nodes suggests that DNA methylation modifications in PCDH10 might be an early event in lymphomagenesis (Table 1). To examine if methylation is uniformly present across the two CGIs or is restricted to specific regions within a CGI, we compared the patterns of MSP of the four regions 
studied in each specific subtype of lymphoma. This analysis revealed that promoter methylation was restricted to smaller segments in CGIs in cases of CLL and MM compared with extensive methylation in other hisotologic subtypes (Supporting Information Fig. 1B).

\section{Validation of Promoter Methylation by Quantitative High-Throughput MALDI-TOF MS Analysis}

To validate the MSP data and for quantitative assessment of methylation, we analyzed 196 samples (20 RL/FH, 12 BL, 25 FL, 26 DLBCL, 38 MZL, 13 MCL, 27 MM, 14 CLL, and 21 T-/NK cell) that were also analyzed by MSP, utilizing high throughput MALDI-TOF MS methylation analysis (Sequenom). For this analysis we chose CpG Island 2 (CGI-2) covering $78 \mathrm{CpG}$ sites in three amplicons (Supporting Information Fig. 1A). Of these, $47 \mathrm{CpG}$ sites, which include single $\mathrm{CpG}$ sites and multiple $\mathrm{CpGs}$ that fall in one fragment, were considered informative after MALDI-TOF MS read out and quality control analysis. Methylation fraction per $\mathrm{CpG}$ was calculated as described previously (Narayan et al., 2011). Mean \pm SEM was $0.2018 \pm 0.03$ for $\mathrm{RL} / \mathrm{FH}$ specimens, $0.2293 \pm 0.03$ for CLL, 0.2082 \pm 0.03 for MM, $0.3193 \pm 0.05$ for MCL, $0.4109 \pm 0.04$ for DLBCL, $0.5483 \pm 0.04$ for $\mathrm{BL}, 0.3565 \pm 0.03$ for $\mathrm{FL}$, $0.3486 \pm 0.02$ for MZL, and $0.3094 \pm 0.04$ for T-/NK cell cases (Fig. 1A). Differences in methylation levels between RL/FH samples and CLL $(P=0.46)$ or MM $(P=0.88)$ were not statistically significant, while other histologic subtypes (BL, DLBCL, FL, MZL, MCL, and T-NHL) showed significant differences compared with $\mathrm{RL} / \mathrm{FH}$ samples (Fig. 1A). The levels of methylation were highest in $\mathrm{BL}$ samples $(P=0.0001)$ among all the histologic subtypes (Figs. 1A and 1B). Supervised one-way hierarchical cluster analysis of $\mathrm{RL} / \mathrm{FH}$ samples in combination with each histologic subtype of NHL cases resulted in two or more major clusters depending on the levels of methylation (Fig. 1B, Supporting Information Fig. 2). In the present analysis, we classified the top two clusters as clusters 1 and 2, which contained cases harboring the highest levels of methylation, and the remaining cases into one group. This analysis showed that all the 12 cases of BL were present in cluster 1 or 2 , while only 4 of the 20 samples of $\mathrm{RL} / \mathrm{FH}$ were present in clusters 1 and 2. These data suggest that $\mathrm{PCDH} 10$ promoter methylation is high in $\mathrm{BL}$ samples as compared with $\mathrm{RL} / \mathrm{FH}$ samples (Fig. 1B, top panel). Similarly, a large majority of samples from DLBCL, FL, MZL, MCL, and T-NHL were grouped in cluster 1 or 2 along with 3 to 4 samples of RL (Fig. 1 and Supporting Information Fig. 2). Nonsupervised cluster analysis of all the samples together further confirmed these findings where the same four RL/ FH samples were grouped with highest methylated lymphoma cases while the remaining $16 \mathrm{RL} /$ FH cases clustered with samples harboring low methylation (Supporting Information Fig. 3). On the other hand, only few samples from MM and CLL appeared in cluster 1 and 2 along with three to four samples of RL/FH (Supporting Information Fig. 2). This observation is consistent with the MSP data showing localized methylation in CLL and MM samples. These data further suggest that the PCDH10 promoter methylation levels vary in different histologic subtypes of NHL.

\section{PCDHIO Promoter Methylation Occurs Early in Lymphomagenesis}

As shown above, $29 \%$ of lymph nodes exhibiting $\mathrm{RL} / \mathrm{FH}$ displayed promoter hypermethylation by MSP compared with $>50 \%$ of cases in NHL histologic subtypes and multiple myeloma (Table 1). Similarly, Sequenom methylation analysis showed 0.2018 fraction of $\mathrm{CpG}$ methylation in $\mathrm{RL} / \mathrm{FH}$ lymph nodes. This level of methylation in RL/FH is lower than most histologic subtypes of mature lymphomas (Fig. 1 and Supporting Information Fig. 2) and higher than normal bone marrow samples $(3.6 \%$ by MSP and 0.115 methylation fraction by Sequenom analysis) as reported previously (Narayan et al., 2011). These data suggest that PCDH10 promoter methylation occurs early in lymphoma development. As stated above, hierarchical cluster analysis of Sequenom MALDITOF MS methylation data identified the same 4 of $20 \mathrm{RL} / \mathrm{FH}$ samples clustered with several histologic subtypes of lymphoma samples exhibiting higher methylation (Fig. 1B and Supporting Information Figs. 2 and 3). This observation implies that these 4 reactive lymph nodes harbor $P C D H 10$ promoter methylation similar to high-grade lymphomas such as BL, DLBCL and MZL.

Of the $4 \mathrm{RL} / \mathrm{FH}$ samples that showed higher methylation, 2 specimens were obtained from the same patient's adenoids and tonsils with previous history of liver transplant and exhibited florid follicular hyperplasia, without any evidence of NHL. These 2 samples consistently grouped in cluster 1 or 2 by nonsupervised analysis. The adenoid 


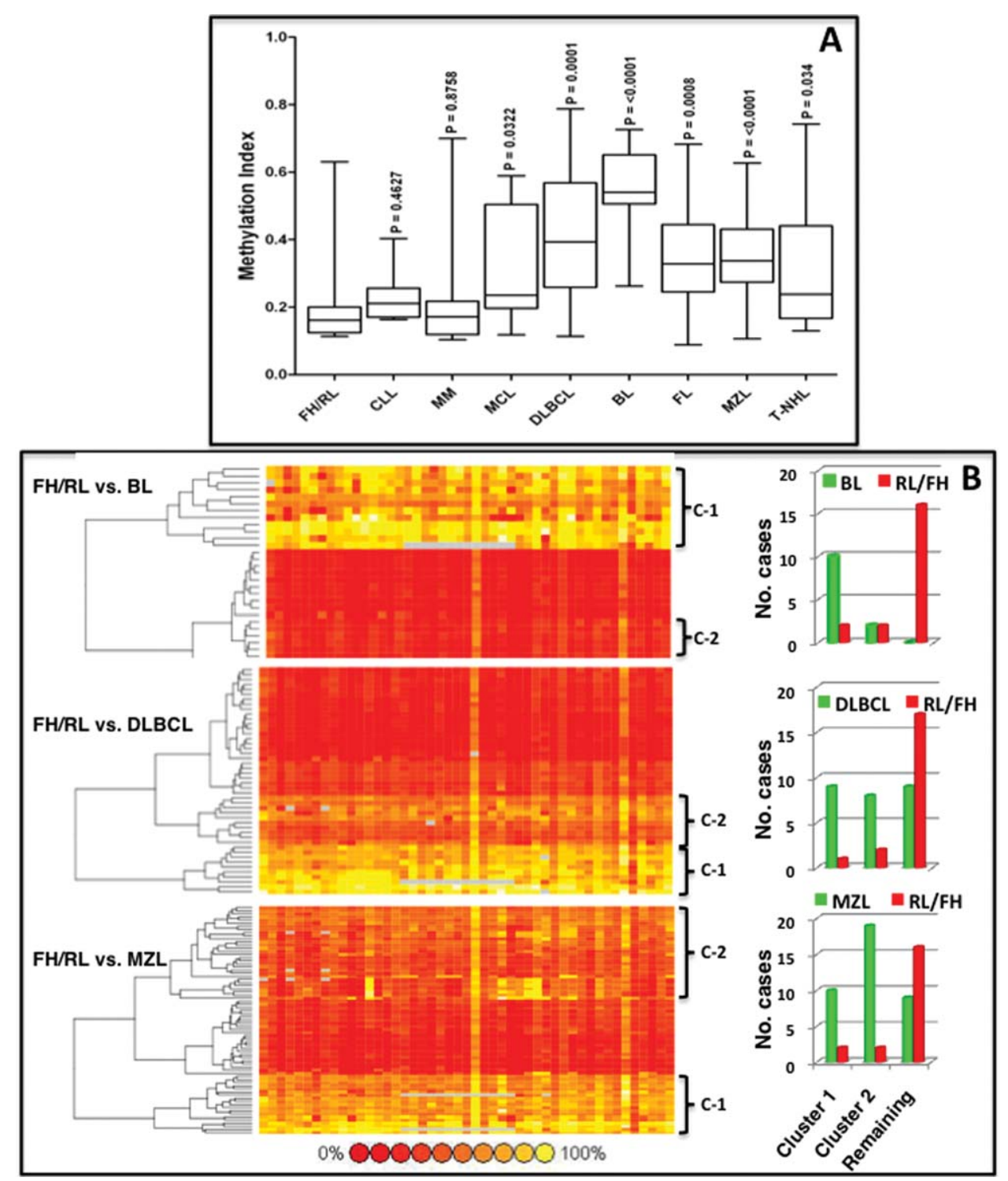

Figure I. Quantitative analysis of methylation of $P C D H I O$ promoter region in reactive lymphoid hyperplasia and various subtypes of lymphoma using Sequenom EpiTYPER. (A). Box-plot showing methylation fraction in various lymphoma subtypes. Significant differences between $\mathrm{RL} / \mathrm{FH}$ and histologic subtype of lymphoma are shown by $P$ value. Box plot shows median, 25th and 75th percentile, minimum and maximum values. (B). Heat maps showing one dimensional cluster analysis of methylation data generated by Sequenom's MassArray and EpiTyper (left panel) between reactive lymph nodes analyzed with $B L, D L B C L$, or MZL. Histogram shows number of cases present in each cluster

sample showed a mean methylation fraction of 0.63 and the tonsil 0.45 by Sequenom methylation analysis, which is similar to the methylation levels seen in high-grade lymphomas (Fig. 1B and Supporting Information Figs. 2 and 3). Karyotype analysis identified the same chromosomal translocation $[\mathrm{t}(1 ; 14)(\mathrm{q} 21 ; \mathrm{q} 32)]$ in both adenoids and tonsils with the adenoids demonstrating a nonclonal ( 1 of 41 metaphases) abnormality, while the tonsils (right panel). CpG Units are arranged along the $x$-axis and samples are on the $y$-axis. Right brackets in left panel indicate clusters of methylated cases. Missing data values are annotated in gray. The red and yellow scale at the bottom represents the percentage methylation with dark red as lowest methylation and dark yellow as highest ( 100\%) methylation. BL, Burkitt lymphoma; C, cluster; CLL, chronic lymphocytic leukemia; DLBCL, diffuse large B-cell lymphoma; RL/FH, reactive lymph node/follicular hyperplasia; FL, follicular lymphoma; MCL, mantle cell lymphoma; MM, multiple myeloma; MZL, marginal zone lymphoma; T-NHL, T-cell Non-Hodgkin lymphoma.

exhibited a minor clone ( 3 of 40 metaphases) harboring this translocation (Fig. 2A). The $\mathrm{t}(1 ; 14)(\mathrm{q} 21 ; \mathrm{q} 32)$ was reported in post-transplant RL/FH (Vakiani et al., 2007) and has been shown to be an early event in lymphomagenesis of mature B-cell lymphomas. The breakpoint at $14 \mathrm{q} 32$ is being the site of the immunoglobulin heavy chain $(I G H)$ (Pinkerton et al.,1992; Dyomin et al., 2000). FISH analysis using IGH break apart 


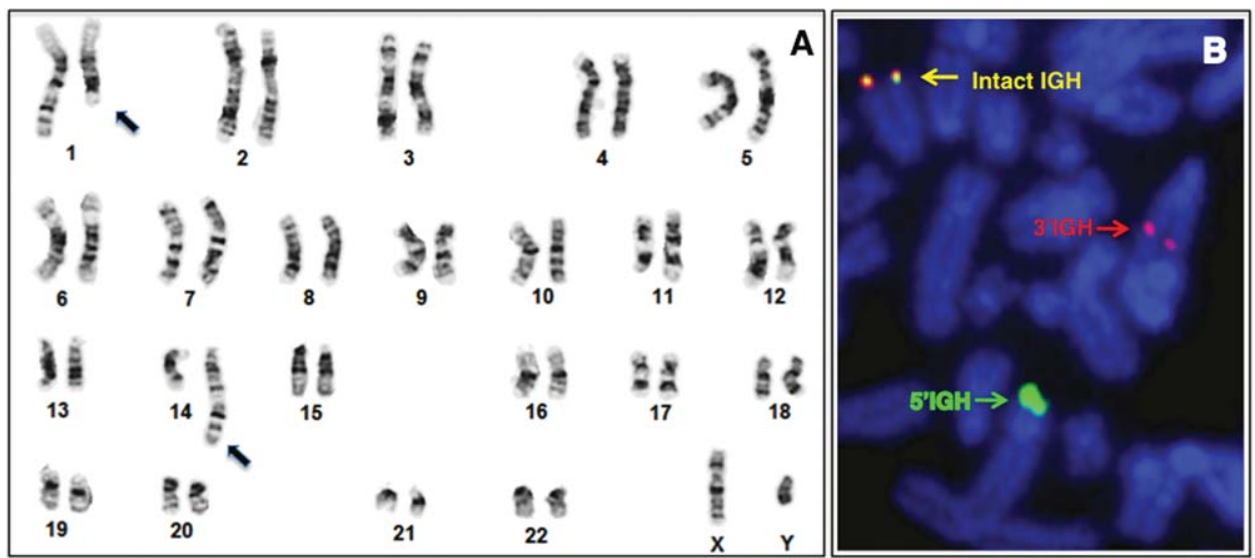

Figure 2. Cytogenetic analysis of a case of tonsils sample diagnosed as follicular hyperplasia and subsequently progressed to post-transplant lymphoproliferative disorder (PTLD) on followup. (A). A G-banded karyotype from tonsils showing $\mathrm{t}(I ; \mid 4)(\mathrm{q} 21 ; \mathrm{q} 32)$ as indicated by arrows. (B). FISH analysis showing IGH rearrangement in PTLD. Yellow arrow indicates intact IGH locus. Red and green arrows indicated rearranged IGH.

TABLE 2. Histologic, Cytogenetic Abnormalities, and Methylation Changes Seen in Reactive Lymph Nodes Exhibiting High Promoter Methylation of PCDHIO

\begin{tabular}{|c|c|c|c|c|c|c|c|c|}
\hline \multirow[b]{2}{*}{ S. No. } & \multirow[b]{2}{*}{ Case } & \multirow[b]{2}{*}{ Tissue } & \multirow[b]{2}{*}{ Diagnosis } & \multicolumn{2}{|c|}{ Methylation } & \multirow[b]{2}{*}{ Karyotype } & \multicolumn{2}{|c|}{$\begin{array}{c}\text { FISH } \\
(\%+v e)\end{array}$} \\
\hline & & & & MSP & MassArray & & IGH & +3 \\
\hline \multirow[t]{4}{*}{ I } & RL305 & Adenoids & $\mathrm{FH}$ & M & 0.63 & $46, X Y, t(I ; \mid 4)(q 21 ; q 32)[1] / 46, X Y[40]$ & 0.8 & ND \\
\hline & RL306 & Tonsils & $\mathrm{FH}$ & M & 0.45 & $46, X Y, t(I ; I 4)(q 2 I ; q 32)[3] / 46, X Y[57]$ & 1.8 & ND \\
\hline & F/U LNI294 & LN-I & PTLD & ND & ND & $46, X Y, t(I ; 14)(q 21 ; q 32)[15] / 47, X Y,+3[5]$ & 20.4 & 16.0 \\
\hline & F/U LNI 295 & LN-2 & PTLD & ND & ND & $\begin{array}{c}46, \mathrm{XY}, \mathrm{t}(\mathrm{I} ; \mathrm{I} \mid 4)(\mathrm{q} 2 \mathrm{I} ; \mathrm{q} 32)[\mathrm{II}] / 47, \mathrm{XY} \\
+3[10] / 46, \mathrm{XY}[2]\end{array}$ & 20.3 & 13.8 \\
\hline 2 & RL575 & LN & $\mathrm{FH}$ & M & 0.32 & $46, X \times[20]$ & 0 & ND \\
\hline 3 & RL7I7 & Tonsil & $\mathrm{FH}$ & M & 0.23 & $\begin{array}{c}46, \mathrm{XY}, \mathrm{t}(5 ; \mathrm{I} 4)(\mathrm{q} 3 \mathrm{I} ; \mathrm{q} 32)[\mathrm{I}] / 47, \mathrm{XY},+\mathrm{i}(\mathrm{I})(\mathrm{q} \mid 0),+ \\
\mathrm{II},-19[\mathrm{I}] / 46, \mathrm{XY}[\mathrm{I8}]\end{array}$ & 0 & ND \\
\hline
\end{tabular}

FISH, fluorescence in situ hybridization; FH, follicular hyperplasia; F/U, follow-up; LN, lymph node; M, methylated; MSP, methylation specific PCR; ND, not done; PTLD, post-transplant lymphoproliferative disorder.

probe (Vysis, Des Plaines, IL) showed low frequency of rearrangement in both tonsils (1.8\%) and adenoids $(0.8 \%)$. After 8 months of follow-up, the patient presented with axillary and cervical lymph node enlargement due to development of a polymorphic post-transplant lymphoproliferative disorder (PTLD), which was associated with $\mathrm{t}(1 ; 14)$ and an additional distinct cytogenetic abnormality (trisomy 3 ) as major clones (Table 2; Supporting Information Fig. 4A). The karyotypic abnormalities were confirmed by FISH using IGH break apart and centromere 3 probes (Vysis) (Fig. 2B and Supporting Information Fig. 4B). The axillary lymph node showed IGH rearrangement in $20.4 \%$ cells and trisomy 3 in $16 \%$ cells, while the cervical lymph node exhibited $20 \%$ cells with IGH rearrangement and trisomy 3 in $14 \%$ cells (Table 2). Methylation analysis on the follow-up
PTLD specimens could not be performed due to lack of tissue availability. This case highlights the fact that the methylation fraction in the RL/FH sample was similar to the level seen in frank lymphomas while the chromosome aberration was presenting a very low frequency that was almost undetectable by FISH analysis. These data therefore suggest that the onset of promoter hypermethylation likely occurred prior to the origin of the chromosome abnormality in this case and indicate that $\mathrm{PCDH} 10$ methylation and potentially other genes might precede chromosomal translocations in the development of lymphoma.

The third RL/FH specimen that showed highlevel of promoter methylation of $P C D H 10$, which occurred in an individual with $\mathrm{FH}$, also demonstrated a nonclonal cytogenetic abnormality by karyotype, while FISH using IGH break apart 


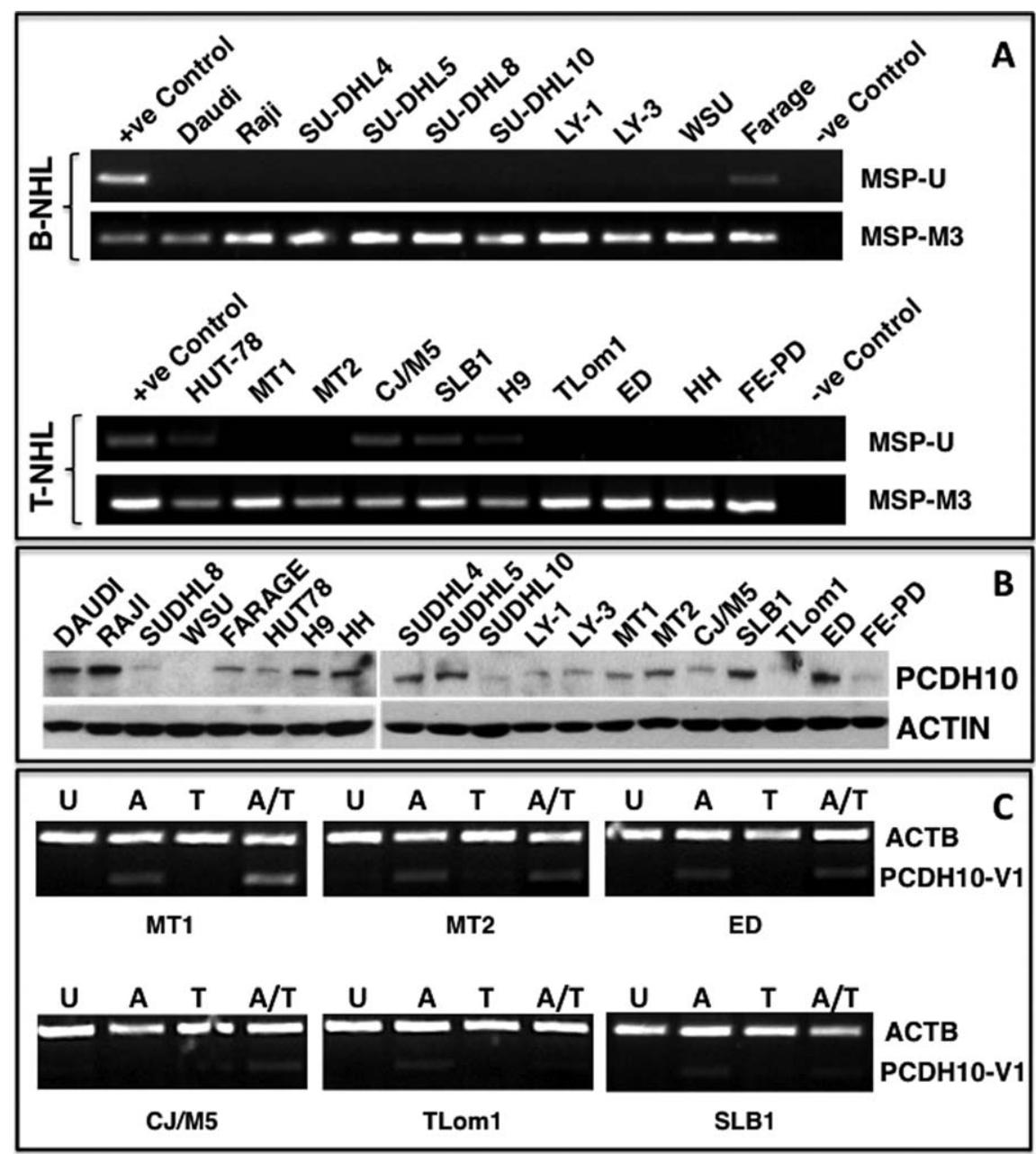

Figure 3. Promoter methylation and expression analysis of PCDHIO in NHL cell lines. (A). Methylation-specific PCR analysis of PCDHIO in B- (top panel) and T- (bottom panel) NHL cell lines. MSP, methylation specific PCR; $U$, unmethylated primer; $M$, methylated primer. (B). Western blot showing PCDHIO protein expression. (C).

probe was negative (Table 2). The fourth specimen with high levels of methylation occurred in an individual with RL and showed neither a karyotypic abnormality nor an IGH rearrangement. No follow-up specimens from these two cases were available. Of note, all the RL/FH samples that showed a high methylation index were also positive by MSP analysis.

\section{PCDHIO Promoter Hypermethylation Correlates with Down-regulated Expression and Pharmacologic Inhibition of DNMTs Reactivates its Expression in NHL Cell Lines}

Since a large proportion of primary B- and T-NHL cases harbored promoter methylation, we examined the status of $\mathrm{PCDH} 10$ promoter hypermethylation in NHL cell lines. We tested 10
Analysis of mRNA expression by RT-PCR analysis to identify the effects of treatment using inhibitors of methylation and HDAC in T-NHL cell lines. $A$, azacytidine; $A C T B$, beta actin; $A / T$, azacytidine/trichostatin; $T$, trichostatin; U, untreated; $\mathrm{VI}$, variant I.

B-NHL cell lines and 10 T-NHL cell lines by the MSP method. All the 20 cell lines exhibited promoter methylation of one or both CGIs. Of the 10 B-NHL cell lines examined, both alleles were methylated in both CGIs in all cell lines except one. The cell line Farage showed presence of both methylated and unmethylated alleles in CGI-2 (Fig. 3A, top panel). Among the T-NHL cell lines, six cell lines (MT1, MT2, ED, TLom1, HH, and FE-PD) showed only methylated alleles in both CGI-1 and CGI-2. The remaining 4 T-NHL cell lines (CJ/M5, SLB1, H9, and HUT-78) showed both methylated and unmethylated alleles in CGI1 or CGI-2 (Fig. 3A, bottom panel). The cell lines H9 and HUT-78 exhibited both CGIs with heterozygous methylation, while the cell lines CJ/M5 and SLB1 showed unmethylated CGI-1 and heterozygous methylation in CGI-2. 
To examine the transcription levels of PCHD10 in NHL cell lines, we performed RTPCR analysis of two PCDH10 transcript variants (Variant 1 spanning exons 1 and 3; Variant 2 with a single exon) on all cell lines. Since variant 2 is a single intronless exon, RNA isolated from the cell lines was treated with DNase to avoid DNA amplification. All the $10 \mathrm{~B}-\mathrm{NHL}$ cell lines examined failed to show any detectable levels of expression of variant 1 , while the variant 2 was detected in three cell lines (Raji, SUDHL-5, and LY-3). Of the 10 T-cell NHL cell lines, three (MT1, TLom1, and HH) showed no detectable expression of both variants. Detectable transcripts of only variant 2 were found in four cell lines (MT2, CJ/M5, ED, and SLB1) while variant 1 was undetectable. The remaining three cell lines (H9, HUT-78, and FE-PD) showed detectable levels of expression of both variants. Western blot analysis further confirmed that the protein levels of PCDH10 were lacking or downregulated in all B- and T-NHL cell lines compared with actin control (Fig 3B). Sixteen cell lines showed very low expression, while the remaining four cell lines (Daudi, Raji, SLB1, and ED) showed relatively higher expression. These data support the notion that $P C D H 10$ expression is down regulated in relation to promoter hypermethylation in the majority of NHL cell lines. The cell line data combined with the data on primary NHL specimens confirm that $P C D H 10$ promoter hypermethylation is a common phenomenon in mature lymphoma and multiple myeloma.

Since down-regulated or complete lack of expression of $P C D H 10$ variant 1 was found in 17 of 20 NHL cell lines, we treated six T-NHL cell lines (MT1, MT2, CJ/M5, ED, TLom1, and SLB1) by the DNA demethylating agent 5-azaCdR and the HDAC inhibitor TSA to test the reactivation of variant 1 . Semi-quantitative RTPCR analysis exhibited reactivation of the PCDH10 gene after 5-aza-CdR alone or in combination with TSA in all six cell lines (Fig. 3C). All these cell lines showed PCDH10 methylation in one or both alleles. None of the treatments had any effect on the level of expression of the control gene (Fig. 3C). Thus, these data establish that demethylation of the PCDH10 promoter effectively reactivates gene expression by reversing the effect of methylation in cell lines. Overall, these data demonstrate that $P C D H 10$ down regulation in the majority of NHL cell lines is a consequence of promoter methylation.

\section{PCDHIO Inactivation Results in Increased Resistance to Doxorubicin in NHL Cell Lines}

Since the DNA promoter methylation of specific genes plays a significant role in tumorigenic processes and response to treatment, we examined the effects of $P C D H 10$ promoter methylation and its associated gene silencing on treatment response using a panel of NHL cell lines after exposure to commonly used drugs for the treatment of lymphoid malignancies. We tested all 20 NHL cell lines for cytotoxicity by MTT assay and analyzed the data on the basis of methylation of one or both alleles to defined doses of doxorubicin, dexamethasone, bortezomib, methotrexate, and L-asparaginase. Since all NHL cell lines showed either one or both copies methylated, we used two unmethylated leukemia cell lines (MOLT16 and T-ALL) as controls (Narayan et al., 2011). The only drug that showed differential cytotoxicity based on the promoter methylation status was doxorubicin (Figs. 4A and 4B). Other drugs did not exhibit detectable differences in cytotoxicity based on methylation status (Supporting Information Figs. 5A-5D). Of the 10 B-NHL cell lines tested, nine had homozygous methylation and one cell line (Farage) showed both methylated and unmethylated alleles. The nine B-NHL cell lines with homozygous methylation showed significantly lower cytotoxic affects compared with the cell line (Farage) that showed heterozygous methylation $(P=0.009)$. Both groups of B-NHL cell lines were more resistant to doxorubicin treatment compared with unmethylated leukemia cell lines (Fig. 4A). Of the 10 T-NHL cell lines tested, six cell lines showed homozygous methylation and the other four cell lines showed heterozygous methylation. Similar to the B-NHL cell lines, the T-NHL cell lines with both methylated alleles were significantly resistant to doxorubicin compared with the heterozygous methylated cell lines $(P=0.002)$ and unmethylated leukemia cell lines (Fig. 4B). A similar trend for doxorubicin resistance was found when the cell lines were analyzed based on detectable transcripts of $P C D H 10$ gene. Of the nine B-NHL cell lines with complete methylation, three cell lines (Raji, SUDHL-5, and LY-3) showing detectable levels of PCDH10 transcripts were significantly $(P=0.009)$ sensitive to doxorubicin treatment compared with six cell lines that showed no detectable levels of expression (Fig. 4C). Similarly, seven T-NHL cell lines (MT2, CJ/M5, ED, SLB1, H9, HUT-78, and FEPD) that showed detectable levels of variant 1 or 2 were more sensitive to doxorubicin treatment 

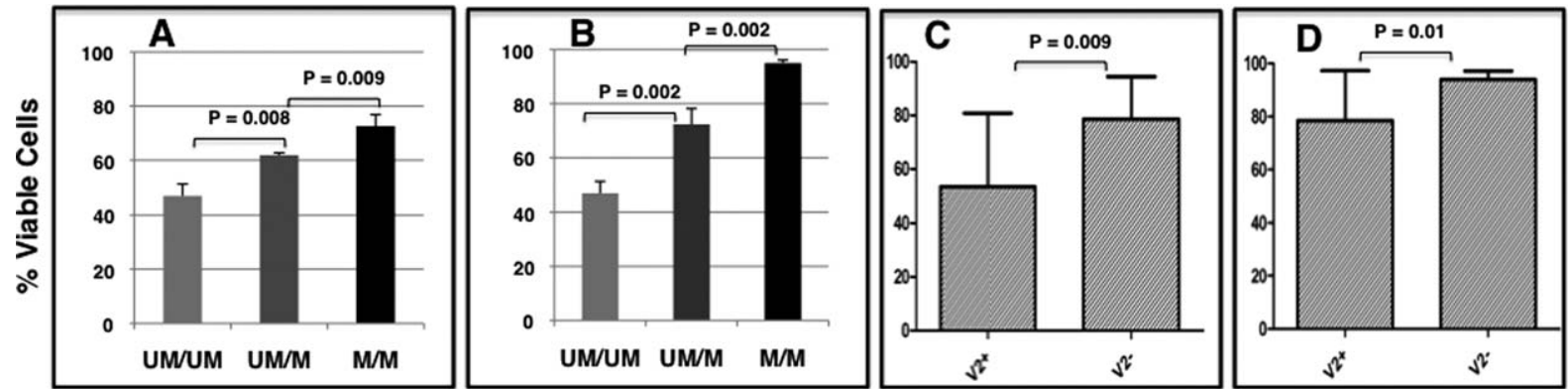

Figure 4. Analysis of cell cytotoxicity in NHL cell lines exposed to leukemic drugs in relation to methylation and silencing of $P C D H I O$ gene. Viability assay showing cell survival in $\mathrm{B}-\mathrm{NHL}(\mathrm{A})$ and $\mathrm{T}-\mathrm{NHL}$ cell lines (B) in relation to methylation status. Viability assay showing cell survival in B-NHL cell lines (C) and T-NHL cell lines (D) in relation to variant 2 mRNA expression. $P$ values are shown above each comparison. UM/UM, both alleles unmethylated in leukemia cell lines; UM/M, both unmethylated and methylated alleles present; $M / M$, both alleles are methylated; V2-, no expression of variant 2; V2+, detectable levels of variant 2 expression.

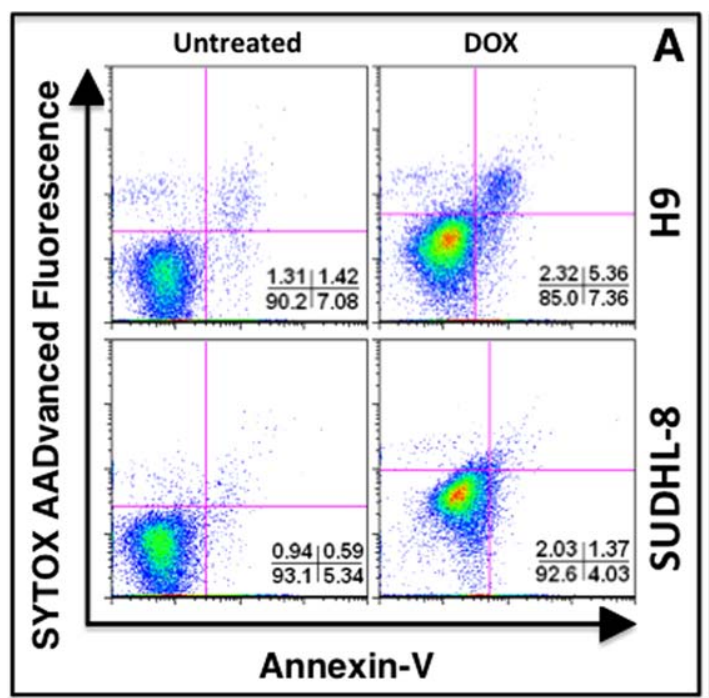

Figure 5. Doxorubicin response to cell death by apoptosis analysis in $\mathrm{NHL}$ cell lines in relation epigenetic silencing of $\mathrm{PCDHIO}$. (A). Flow cytometric analysis of two cell lines showing high frequency of cell death in PCDHIO expressed (H9) cell line compared with nonexpressed cell line (SUDHL-8). (B). Histogram showing significant differ-

compared with three cell lines (MT1, TLom1, and $\mathrm{HH})$ that did not express PCDH10 $(P=0.01)$ (Fig. 4D). Similar to methylation, other drugs did not exhibit detectable differences in cytotoxicity based on expression levels (Supporting Information Figs. 5E-5H). These data suggest that PCDH10 transcriptional down-regulation has a causal affect on doxorubicin-induced cell lethality in NHL cell lines.

We next asked if a similar difference in resistance could be seen in the apoptotic response to doxorubicin treatment. To examine this, we tested two cell lines (H9 and HUT-78) expressing both variants and two cell lines (SUDHL-8 and WSU) with undetectable expression (Fig. 5A). The former had higher fraction of apoptotic cells com-

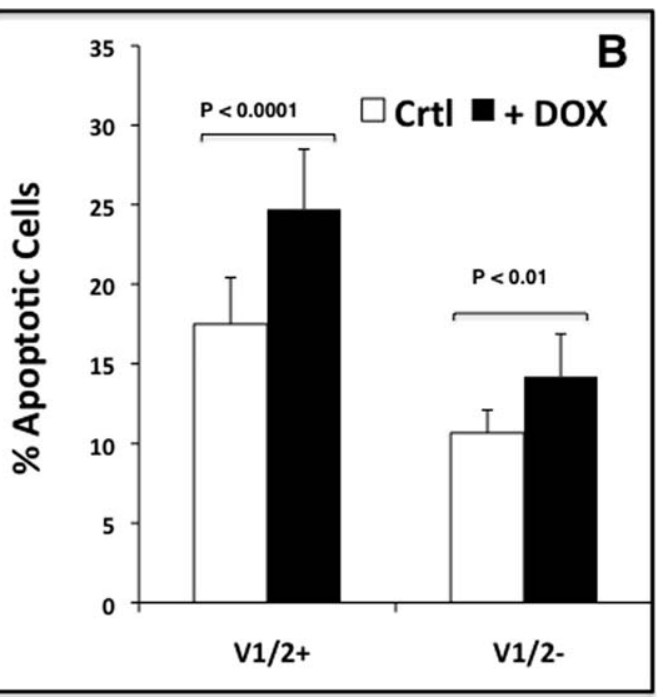

ences cell death response to doxorubicin treatment in $\mathrm{PCDH} / 0$ expressing cell lines compared with nonexpresser cell lines. Ctrl, Control; DOX, doxorubicin; VI/2, variant I and 2. [Color figure can be viewed in the online issue, which is available at wileyonlinelibrary.com.]

pared with nonexpressor cell lines after treatment with doxorubicin (Fig. 5B). To examine further the relationship between methylation status on apoptotic response, we tested all the five drugs on 11 to 17 NHL cell lines. We found significantly reduced apoptosis in all methylated NHL cell lines compared with unmethylated leukemia cell lines (Fig. 6). Also NHL cell lines with homozygous methylation were significantly resistant to apoptosis induction to dexamathasone and methotrexate treatments compared with the cell lines that showed heterozygous methylation (Figs. 6B and 6D). However, other drugs did not show significant difference in apoptotic response based on heterozygous and homozygous methylation status (Figs. 6A, 6C, and 6E). These data suggest 


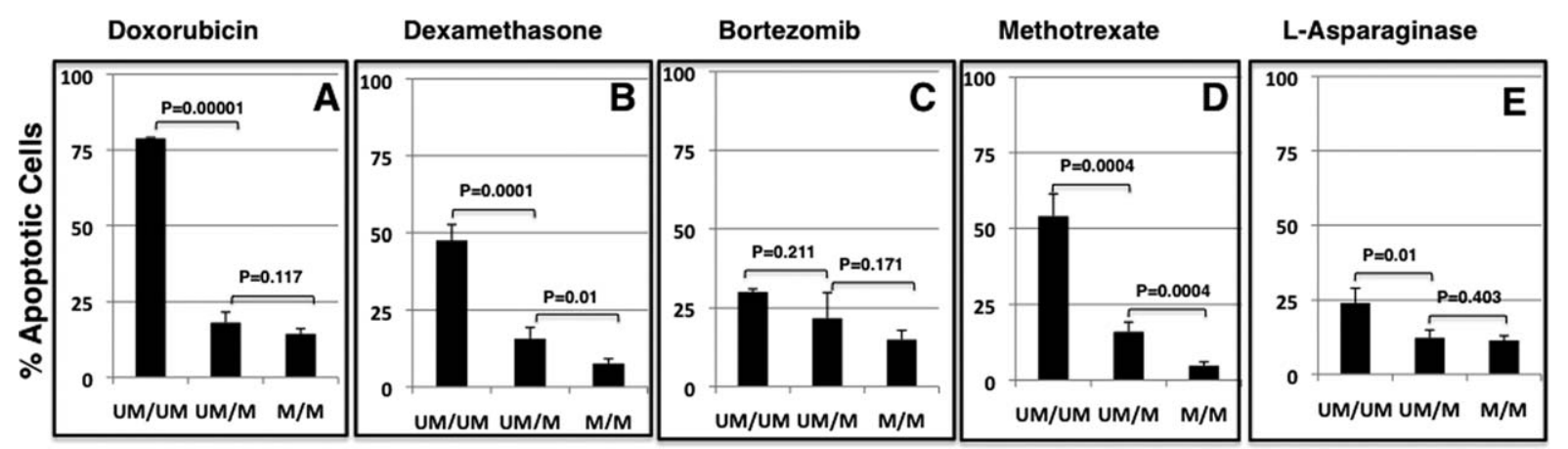

Figure 6. Flow cytomety analysis of apoptotic response in NHL (both $\mathrm{B}$ - and $\mathrm{T}-$ ) cell lines exposed to leukemic drugs in relation to methylation of $\mathrm{PCDH} / 0$ gene. Histograms showing apoptotic response after exposure to doxorubicin (A, 17 cell lines), dexamethasone (B, I5 cell lines), bortezomib ( $C, 15$ cell lines), methotrexate (D, II cell

significantly reduced apoptotic response in PCDH10 inactivated cell lines. Together, our data demonstrate that methylation-mediated inactivation of $\mathrm{PCDH} 10$ negatively influences cellular response to doxorubicin treatment and possibly other drugs in NHL cell lines.

\section{DISCUSSION}

Cadherins play an important role in tumor progression by functioning as suppressors of invasion and metastasis (Jeanes et al., 2008). The PCDH10 gene has shown to be inactivated by promoter methylation in a variety of tumors (Waha et al., 2005; Imoto et al., 2006; Yu et al., 2008, 2009; Narayan et al., 2009, 2011; Cheung et al., 2010). Over expression of this gene significantly inhibits proliferation of solid tumor cells in vitro, supporting its tumor suppressor function (Ying et al., 2006). To examine the role of $\mathrm{PCDH} 10$ in mature lymphoid malignancies, we systematically analyzed qualitative and quantitative promoter hypermethylation in a large cohort of non-Hodgkin lymphomas, including cell lines, and multiple myelomas. Our present finding of high levels of promoter methylation in the vast majority of primary high-grade mature B- and T-cell lymphomas and their derived cell lines substantiate the prior findings that $P C D H 10$ is frequently methylated in different subtypes of malignant lymphomas (Ying et al., 2007). We have previously shown that epigenetic changes in the promoter of $\mathrm{PCDH} 10$ preferentially occur in lymphoid-lineage leukemias compared with myeloid-lineage leukemias (Narayan et al., 2011). The present findings combined with our previous data suggest that $P C D H 10$ methylation is a unique phenomenon in all lymphoid lineage hematologic malignancies. Furthermore, the iden- lines), L-asparaginase ( $E, I 5$ cell lines). $P$ values are shown above each comparison. UM/UM, both alleles unmethylated in leukemia cell lines; $\mathrm{UM} / \mathrm{M}$, both unmethylated and methylated alleles present; $M / M$, both alleles are methylated.

tification of promoter hypermethylation in $29 \%$ of $\mathrm{RL} / \mathrm{FH}$ specimens and evidence of promoter methylation preceding chromosomal translocation in $\mathrm{RL} / \mathrm{FH}$ suggest that inactivation of this gene occurs early and is likely a critical alteration during the neoplastic transformation of mature lymphoid cells.

NHL represents a highly diverse group of malignancies of both B- and T-cell lineages that comprise of different histologic subtypes. The malignant transformation of precursor cells is driven by errors in the machinery that regulates antibody diversification and lineage-specification cause specific chromosomal translocations and oncogenic mutations (Kuppers and Dalla-Favera, 2001). The landscape of genetic lesions in NHL is highly characteristic of specific histologic subtypes and several of the recurrent mutations have been shown to be driver mutations that play a critical role in lymphoma initiation (Shaffer et al., 2012). More recent studies have uncovered structural alterations in genes that regulate chromatin modifications such as BMI1, EZH2, MLL2, CREBBP, and EP300 (Shaffer et al., 2012). The natural course of the different histologic subtypes of NHL is highly variable, ranging from indolent, like in CLL or FL, to highly aggressive, like in BL. The prognosis is also highly variable to current modalities of chemotherapy, radiation and the anti-CD20 antibody (Rituximab) treatment (Shaffer et al., 2012). Although specific genetic changes are diagnostic of specific lymphoma, biomarkers of therapeutic response are lacking in NHL.

DNA methylation and chromatin modifications dictate the biological behavior and response to therapy of cancer cells. Although, in the present study, we identified PCDH10 methylation in 
various NHL histologic subtypes, it was more frequently observed in lymphomas showing evidence of germinal center transit (BL, FL, DLBCL, MZL, and MCL) and T-cell lymphomas compared with nongerminal center origin lymphomas (CLL and MM). Moreover, the levels of promoter methylation were not significantly different among indolent (FL) and aggressive (BL and DLBCL) type lymphomas. Based on these observations, combined with the finding that reactive lymph nodes harboring high-level methylation could progresses to frank lymphoma, we propose a hypothesis that PCDH10 methylation might represent an important early event in lymphomagenesis.

Although the role of PCDH10 in cancer has not been fully elucidated, evidence for the epigenetic silencing of this gene contributing to tumorigenesis has been established (Ying et al., 2006). More recently, $P C D H 10$ was reported to be methylated in early stages of gastric carcinogenesis and its methylation was associated with poor prognosis in gastric cancer patients (Yu et al., 2009). Since $P C D H 10$ is a target of epigenetic silencing in the majority of lymphomas, its implications for chemotherapy response was examined. Although the mechanisms underlying cellular resistance to apoptosis in lymphomas remain unclear, our finding of resistance to cytotoxicity and decreased apoptotic response to doxorubicin treatment suggests that PCDH10 methylation and inactivation in cell lines might play a role in chemotherapy resistance. Doxorubicin, a cellular target of topoisomerase-2 (Top2), is a widely used chemotherapeutic drug in many cancer types, including lymphomas. Doxorubicin binds to both DNA and Top2 forming doxorubicin-DNA adducts that initiates caspase cascade dependent apoptosis (Swift et al., 2006). The biologic basis of PCDH10-related resistance mechanisms and cooperation with other genetic/ epigenetic pathways in chemoresistance of NHL remains to be determined. Ultimately designing successful epigenetic induction therapies similar to myeloid leukemias is a major challenge for therapy involving epigenetic agents in NHL.

In summary, this study has demonstrated the occurrence of epigenetic silencing of PCDH1O in a high proportion of NHL and its likely onset at an early stage of lymphomagenesis suggesting a role for PCDH10 in lymphoma development. The finding of doxorubicin resistance in association with PCDH10 inactivation may have relevance in further exploring the role of epigenetic alterations and ultimately in establishing epigenetic therapies in lymphomas.

\section{REFERENCES}

Baylin SB, Jones PA. 2011. A decade of exploring the cancer epigenome-Biological and translational implications. Nat Rev Cancer 11:726-734.

Cheung HH, Lee TL, Davis AJ, Taft DH, Rennert OM, Chan WY. 2010. Genome-wide DNA methylation profiling reveals novel epigenetically regulated genes and non-coding RNAs in human testicular cancer. Br J Cancer 102:419-427.

Dyomin VG, Palanisamy N, Lloyd KO, Dyomina K, Jhanwar SC, Houldsworth J, Chaganti RS. 2000. MUC1 is activated in a B-cell lymphoma by the $\mathrm{t}(1 ; 14)(\mathrm{q} 21 ; \mathrm{q} 32)$ translocation and is rearranged and amplified in B-cell lymphoma subsets. Blood 95: 2666-2671.

Garcia-Manero G, Yang H, Kuang SQ, O’Brien S, Thomas D, Kantarjian H. 2009. Epigenetics of acute lymphocytic leukemia. Semin Hematol 46:24-32.

Imoto I, Izumi H, Yokoi S, Hosoda H, Shibata T, Hosoda F, Ohki M, Hirohashi S, Inazawa J. 2006. Frequent silencing of the candidate tumor suppressor PCDH20 by epigenetic mechanism in non-small-cell lung cancers. Cancer Res 66:46174626.

Jeanes A, Gottardi CJ, Yap AS. 2008. Cadherins and cancer: how does cadherin dysfunction promote tumor progression? Oncogene 27:6920-6929.

Kuppers R, Dalla-Favera R. 2001. Mechanisms of chromosomal translocations in B cell lymphomas. Oncogene 20:5580-5594.

Li Y, Yang ZS, Song JJ, Liu Q, Chen JB. 2012. Protocadherin-10 is involved in angiogenesis and methylation correlated with multiple myeloma. Int J Mol Med 29:704-710.

Narayan G, Scotto L, Neelakantan V, Kottoor SH, Wong AH, Loke SL, Mansukhani M, Pothuri B, Wright JD, Kaufmann AM, Schneider A, Arias-Pulido H, Tao Q, Murty VV. 2009. Protocadherin PCDH10, involved in tumor progression, is a frequent and early target of promoter hypermethylation in cervical cancer. Genes Chromosomes Cancer 48:983-992.

Narayan G, Freddy AJ, Xie D, Liyanage H, Clark L, Kisselev S, Un Kang J, Nandula SV, McGuinn C, Subramanivam S, Alobeid B, Satwani P, Savage D, Bhagat B, Murty VV. 2011. Promoter methylation-mediated inactivation of PCDH10 in acute lymphoblastic leukemia contributes to chemotherapy resistance. Genes Chromosomes Cancer 50:1043-1053.

Nowak D, Stewart D, Koeffler HP. 2009. Differentiation therapy of leukemia: 3 Decades of development. Blood 113:3655-3665.

Pinkerton PH, Reis MD, DeCoteau J, Srigley JR, Dube ID, London B. 1992. A lineage-specific $\mathrm{t}(1 ; 14)(\mathrm{q} 21 ; \mathrm{q} 32)$ as an early event in development of B-cell clonal expansion. Cancer Genet Cytogenet 64:166-169.

Seton-Rogers S. 2012. Lymphoma: Epigenetic therapy gains momentum. Nat Rev Cancer 12:798-799.

Shaffer AL, 3rd, Young RM, Staudt LM. 2012. Pathogenesis of human B cell lymphomas. Annu Rev Immunol 30:565-610.

Swerdlow SH CE, Harris NL, Jaffe ES, Pileri SA, Stein H, Thiele J, Vardiman JW. 2008. WHO Classification of Tumours of Haematopoietic and Lymphoid Tissues, 4th ed. Lyon, France: International Agency for Research on Cancer. pp. 180319.

Swift LP, Rephaeli A, Nudelman A, Phillips DR, Cutts SM. 2006. Doxorubicin-DNA adducts induce a non-topoisomerase IImediated form of cell death. Cancer Res 66:4863-4871.

Vakiani E, Nandula SV, Subramaniyam S, Keller CE, Alobeid B, Murty VV, Bhagat G. 2007. Cytogenetic analysis of B-cell posttransplant lymphoproliferations validates the World Health Organization classification and suggests inclusion of florid follicular hyperplasia as a precursor lesion. Hum Pathol 38:315325.

Waha A, Guntner S, Huang TH, Yan PS, Arslan B, Pietsch T, Wiestler OD. 2005. Epigenetic silencing of the protocadherin family member PCDH-gamma-A11 in astrocytomas. Neoplasia 7:193-199.

Yagi T. 2008. Clustered protocadherin family. Dev Growth Differ 50(Suppl 1):S131-S140.

Ying J, Li H, Seng TJ, Langford C, Srivastava G, Tsao SW, Putti T, Murray P, Chan AT, Tao Q. 2006. Functional epigenetics identifies a protocadherin PCDH10 as a candidate tumor suppressor for nasopharyngeal, esophageal and multiple other carcinomas with frequent methylation. Oncogene 25:1070-1080.

Ying J, Gao Z, Li H, Srivastava G, Murray PG, Goh HK, Lim CY, Wang Y, Marafioti T, Mason DY, Ambinder RF, Chan AT, Tao Q. 2007. Frequent epigenetic silencing of protocadherin 10 by 
methylation in multiple haematologic malignancies. $\mathrm{Br} \mathrm{J}$ Haematol 136:829-832.

Yu J, Cheng YY, Tao Q, Cheung KF, Lam CN, Geng H, Tian LW, Wong YP, Tong JH, Ying JM, Jin H, To KF, Chan FK, Sung JJ. 2009. Methylation of protocadherin 10, a novel tumor suppressor, is associated with poor prognosis in patients with gastric cancer. Gastroenterology 136:640-651.
Yu JS, Koujak S, Nagase S, Li CM, Su T, Wang X, Keniry M, Memeo L, Rojtman A, Mansukhani M, Hibshoosh H, Tycko B, Parsons R. 2008. PCDH8, the human homolog of PAPC, is a candidate tumor suppressor of breast cancer. Oncogene 27: 4657-4665.

Zain J, O'Connor OA. 2010. Targeting histone deacetyalses in the treatment of B- and T-cell malignancies. Invest New Drugs 28(Suppl 1):S58-S78. 